\title{
Dentures: Types, Benefits and Potential Complications
}

\section{Tibor Karoly Fábián, Pal Fejerdy, Peter Hermann}

Publisher: Nova Science Publishers, Inc.

Language: English

ISBN: 978-1-61942-280-3

Edition: $1 / \mathrm{e}$

Publish Year: 2012

Pages: 278, illustrated

Price: $\$ 165.00$

The book entitled Dentures: Types, Benefits and Potential Complications is well structured over its thirteen chapters and is an overview of the restoration possibilities in the field of prosthetic dentistry, representing the vision of the Semmelweis University Budapest.

Starting from the personal "prosthetic classification" (Fábián and Fejérdy, 1979) the authors develop a classification to establish a plan for oral rehabilitation and procedures to be performed so as to fulfill this plan.

The authors also define the importance of preprosthetic treatment and interdisciplinary collaboration. Most often the success of prosthetic treatment of adult patients is conditioned by the integration of orthodontic treatment and multidisciplinary approach.

The book also tackles the masticatory function and congnitive ability, the different possibilities of restoring the edentulous (inlay retained fixed partial dentures, removable partial dentures with double crowns and complete dentures), clinical decisions in the edentulous rehabilitation in developing countries. The use of denture adhesives rejects some of the myths related to adhesives in order

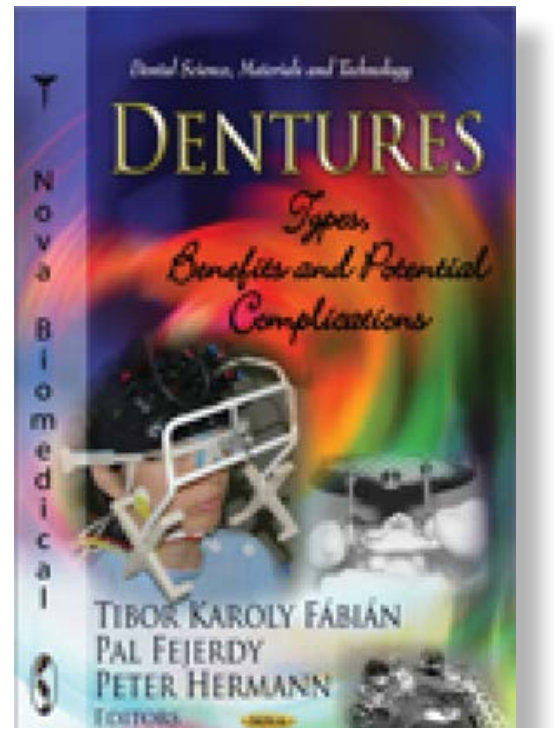

to allow good use and avoid misuse of the same. At the same time the authors highlight the importance of oral hygiene in denture wearers. Continuous swallowing or aspiration of microorganisms from denture plaque exposes elderly patients to the risk of unexpected infections. The frequency of the psychogenic symptoms induced by dentures is also reported and also how to prevent and treat them.

The final chapter focuses on scientific data about bone formation around dental implants using Pulsed Electromagnetic Field (PEMF) therapy for the clinical practice.

If you are interested in partially and totally edentulous patients and you have frequently encountered such cases, going through this book will provide you with answers to the problems

https://doi.org/10.25241/stomaeduj.2015.2(2).bookreview.1

\footnotetext{
Marian-Vladimir

Constantinescu

Bucharest, Romania
Emal: The Book Review is drafted in the reviwer's sole wording and illustrates his opinions. 